\title{
A RELAÇÃO ENTRE UMA PRODUÇÃO MAIS LIMPA E O SETOR TERCIÁRIO: UM ESTUDO FUNDAMENTADO NAS DIMENSÕES DA QUALIDADE EM SERVIÇOS
}

\author{
THE RELATIONSHIP BETWEEN CLEANER \\ PRODUCTION AND TERTIARY SECTOR: A STUDY BASED \\ IN SERVICE QUALITY DIMENSIONS
}

Data de submissão: 07-12-2014

Aceite: $10-08-2015$

Bernardo Dias Machado ${ }^{1}$

Martiele Cortes Borges ${ }^{2}$

Bruno Anicet Bittencourt ${ }^{3}$

\section{RESUMO}

O setor de serviços tem se tornado cada vez mais atuante no cenário mundial, representando hoje mais de $60 \%$ do Produto Interno Bruto (PIB) brasileiro e aproximadamente $80 \%$ do PIB de países desenvolvidos. Além disso, a preocupação do setor com as questões ambientais vem ganhando significativo espaço, principalmente pela expansão da consciência coletiva relacionada ao meio ambiente. Por isso, essas empresas necessitam reestruturar suas práticas e adotarem um novo posicionamento em face desse tema. Nesse sentido, uma alternativa é a aplicação contínua de uma estratégia ambiental preventiva integrada aos processos que aumente a eficiência e, ao mesmo tempo, reduza os riscos ao meio ambiente: a Produção Mais Limpa $(\mathrm{P}+\mathrm{L})$. Baseando-se nesse contexto, a presente pesquisa tem como objetivo identificar de que forma ocorre a relação entre $\mathrm{P}+\mathrm{L}$ e o setor de serviços. Para isso, será apresentada uma associação, baseada na literatura, entre a metodologia de implantação da $P+L$ e as cinco dimensões da qualidade utilizadas pelo setor de serviços como forma de verificar os impactos na percepção dos clientes quanto a melhorias nas operações e nos processos. O principal resultado indica que a relação existe, pois foram identificadas várias interfaces e impactos da aplicação da $\mathrm{P}+\mathrm{L}$ nas dimensões da qualidade em serviços percebidos pelos clientes.

Palavras-chave: Produção Mais Limpa $(P+L)$; setor de serviços; dimensões da qualidade em serviços

${ }^{1}$ Possui graduação em Administração pela Universidade Federal do Rio Grande do Sul - UFRGS, graduação em andamento em Engenharia de Produção pela Universidade Federal do Pampa - UNIPAMPA e mestrado em andamento em Administração pela Universidade Federal do Rio Grande do Sul - UFRGS. Porto Alegre. Rio Grande do Sul. Brasil. E-mail: bernardo.machado@ufrgs.br

${ }^{2}$ Possui graduação em Administração pela Universidade Federal do Rio Grande do Sul - UFRGS e mestrado em andamento em Agronegócios pela Universidade Federal do Rio Grande do Sul - UFRGS. Porto Alegre. Rio Grande do Sul. Brasil. E-mail: martieleborges@ gmail.com

${ }^{3}$ Possui graduação em Administração pela Universidade Federal do Rio Grande do Sul - UFRGS e mestrado em andamento em Administração pela Universidade Federal do Rio Grande do Sul - UFRGS. Porto Alegre. Rio Grande do Sul. Brasil. E-mail: brunoabittencourt@gmail.com 


\begin{abstract}
The service sector has become increasingly active on the world stage, today representing over $60 \%$ of Brazil's Gross Domestic Product (GDP) and about $80 \%$ of GDP in developed countries. Moreover, the concern of the sector with environmental issues has gained significant space, mainly by the expansion of collective consciousness related environment. Therefore, these companies need to restructure their practices and adopt a new position in the face of such issues. Accordingly, an alternative is the continuous application of an integrated preventive environmental strategy to processes that increase efficiency while reducing risks to the environment: Cleaner Production (CP). Based on this background, this research aims to identify how is the relationship between $C P$ and the service sector. For that, will be presented an association based on the literature from the methodology of implementation of $C P$ and the five dimensions of quality used by the services sector as a way to verify impacts on customer perception as to improvements in operations and processes will be presented. The main result indicates that the relationship exists, because multiple interfaces and impacts of the implementation of $\mathrm{CP}$ in quality service dimensions perceived by customers were identified.
\end{abstract}

Keywords: Cleaner Production (CP); service sector; service quality dimensions

\title{
1 INTRODUÇÃO
}

O setor de serviços tem se tornado cada vez mais atuante no cenário mundial, representando hoje mais de $60 \%$ do Produto Interno Bruto (PIB) brasileiro e aproximadamente $80 \%$ do PIB de países desenvolvidos (MDIC, 2014). Além disso, a preocupação do setor com as questões ambientais vem ganhando significativo espaço (CNC-SEBRAE, 2008), principalmente pela expansão da consciência coletiva relacionada ao meio ambiente. Por isso, as empresas do setor necessitam reestruturar práticas e adotar um novo posicionamento em face de tais questões (TACHIZAWA, 2004).

Uma alternativa é a aplicação contínua de uma estratégia ambiental preventiva integrada aos processos que aumente a eficiência e reduza os riscos ao meio ambiente: a Produção Mais Limpa (P+L) (UNIDO/UNEP, 1991; UNIDO/UNEP, 1995). Embora a conceituação de P+L, conforme UNIDO/UNEP (1991), incorpore o setor de serviços, pouca atenção é dada na academia para estudos referentes à $\mathrm{P}+\mathrm{L}$ nesse setor, que é debatida e aplicada significativamente pelo setor da manufatura, por exemplo (FRESNER et al., 2010, MASSOTE; SANTI, 2013).

De acordo com Téboul (1999) e Silva e Medeiros (2006), a implementação desse tipo de ferramenta gerencial deve ocorrer de forma diferenciada do que acontece na indústria, devido às peculiaridades inerentes ao setor terciário, como a participação direta do cliente no processo produtivo e o grau de tangibilidade do serviço. Por essa razão, alguns autores sugerem que, para esse setor, deveria ser adotado o nome alternativo "Serviços Mais Limpos" ao invés de P+L (GLAVIC; LUKMAN, 2007).

Diante disso, a presente pesquisa tem como objetivo identificar de que modo ocorre a relação entre $\mathrm{P}+\mathrm{L}$ e o setor de serviços. Para isso, será apresentada uma associação, fundamentada na literatura, entre a metodologia de implantação da $P+L$ (UNEP/UNIDO, 1991) e as cinco dimensões da qualidade de Parasuraman, Zeithaml e Berry (1988), utilizadas pelo setor de serviços como forma de verificar os impactos na percepção dos clientes quanto a melhorias nas operações e nos processos (FITZSIMMONS; FITZSIMONS, 2007).

Este artigo está organizado em quatro seções, além desta introdutória. A seção 2 apresenta a revisão da literatura sobre a $\mathrm{P}+\mathrm{L}$ no setor de serviços e sobre a gestão da qualidade em serviços; a seção 3 explicita o método de pesquisa; a seção 4 discute a análise dos resultados; e a seção 5 discorre acerca das considerações finais. 


\section{REVISÃO DA LITERATURA}

Na presente seção, são apresentados os pressupostos teóricos que embasam esta pesquisa, enfatizando a Produção Mais Limpa $(\mathrm{P}+\mathrm{L})$ no setor terciário e a gestão da qualidade em serviços.

\subsection{A produção mais limpa no setor terciário}

$\mathrm{O}$ conceito de $\mathrm{P}+\mathrm{L}$ apresenta-se como uma estratégia de melhoria ambiental com foco na redução de desperdícios e, consequentemente, na redução de custos organizacionais (GLAVIC; LUKMAN, 2007). Esse programa tem sido difundido especialmente pelo Programa das Nações Unidas para o Ambiente (UNEP) e pela Organização das Nações Unidas para o Desenvolvimento Industrial (UNIDO), que apresentam e definem a $\mathrm{P}+\mathrm{L}$ como uma contínua aplicação nas empresas de estratégia ambiental preventiva a fim de aumentar a ecoeficiência e reduzir os riscos aos seres humanos e ao meio ambiente (UNIDO/UNEP, 1991; UNIDO/UNEP, 1995; CETESB/UNEP, 2002; FRESNER et al., 2010, MASSOTE; SANTI, 2013).

Essa proposta, conforme os dois órgãos, pode ser aplicada nos processos de qualquer setor da economia (UNIDO/UNEP, 1991; UNIDO/UNEP, 1995; CETESB/UNEP, 2002). Porém, ao mesmo tempo em que se inclui o setor terciário no conceito de $\mathrm{P}+\mathrm{L}$, ele é excluído (GLAVIC; LUKMAN, 2007), uma vez que o termo "produção" é compreendido como manufatura e produto (MICHAELIS, 2009) e o termo "serviço" como a atividade de fazer algo para um cliente, ao invés de produzir um bem (CAMBRIDGE, 2013). Por essa razão, alguns autores sugerem uma adaptação da nomenclatura para esse setor: "serviços mais limpos" (GLAVIC; LUKMAN, 2007).

Como consequência de o foco conceitual estar na manufatura, é perceptível a pouca atenção dada aos serviços em pesquisas acadêmicas relativas à $P+L$ (SILVA; MEDEIROS, 2006). Nesse sentido, é relevante compreender que a implantação de ferramentas gerenciais na prestação de serviços, a exemplo da $\mathrm{P}+\mathrm{L}$, deve ocorrer de forma diferenciada devido às suas características, que são distintas da manufatura (SILVA; MEDEIROS, 2006). O conhecimento dessas características peculiares possibilita a otimização no gerenciamento dos serviços e, com isso, uma maior eficiência no uso de programas como a P+L. Aqui se enquadram, por exemplo, fatores como a intangibilidade e o consumo instantâneo pelo cliente (FITZSIMMONS; FITZSIMONS, 2007).

$A \mathrm{P}+\mathrm{L}$ é, assim, uma alternativa que deve ser considerada para a área de serviços (SILVA; MEDEIROS, 2006), apresentando-se como uma abordagem abrangente, integrada e sistêmica, pois inclui mudanças em todos os aspectos organizacionais (SILVA et al., 2013). Esse fato permite que sejam incorporados à empresa do setor terciário conceitos ligados à diminuição da produção de resíduos pelo uso mais racional dos recursos naturais, proporcionando, assim, oportunidades de crescimento fundamentadas em práticas ambientais (SILVA; MEDEIROS, 2006). Evidencia-se, com isso, a relação direta entre a $\mathrm{P}+\mathrm{L}$ e a melhoria contínua das práticas organizacionais na prestação de serviços, objetivo este também da Gestão da Qualidade (SILVA et al., 2013), principal razão pela qual a $\mathrm{P}+\mathrm{L}$ é conceito fundamental para este trabalho.

\subsection{Gestão da qualidade em serviços}

Uma firma que deseja sobreviver e ter sucesso no mercado deve considerar o oferecimento de serviços de qualidade como estratégia essencial (PARASURAMAN, ZEITHAML; BERRY, 1988). Por essa razão, prestadores de serviços orientam-se cada vez mais para a qualidade e, assim, alcançam níveis altos de satisfação de seus clientes (BHAT, 2012). 
A conceituação de qualidade em serviços, conforme Reynolds e Smith (2010), está relacionada a uma avaliação feita pelo consumidor que inclui uma comparação entre as expectativas esperadas e a percepção sobre o serviço prestado. A expectativa está relacionada ao nível do serviço que o cliente espera receber antes de comprá-lo, enquanto que a percepção refere-se ao durante ou após a prestação do serviço. Assim, a diferença entre o esperado e o percebido é a medida da qualidade, podendo, portanto, a satisfação ser positiva ou negativa (FITZSIMMONS; FITZSIMMONS, 2007).

É relevante destacar que a avaliação da qualidade em serviços surge durante o seu processo de prestação. $O$ julgamento realizado pelos clientes é baseado em cinco dimensões: confiabilidade, responsividade, segurança, empatia e aspectos tangíveis. Cada uma das dimensões, de acordo com Parasuraman, Zeithaml e Berry (1988), Fitzsimmons e Fitzsimmons (2007) e Agarwal, Malhotra e Bolton (2010), é definida como:

- $\quad$ confiabilidade - a prestação do serviço prometido de forma precisa e exata, ou seja, a expectativa do cliente quanto ao desempenho confiável de um serviço (este deve ser cumprido no prazo, sem erros ou modificações);

- $\quad$ responsividade (capacidade de resposta) - a disposição dos funcionários para o auxílio do cliente e para o fornecimento rápido de ajuda. $O$ fato de deixar o cliente esperando origina uma percepção negativa no que se refere à qualidade. Caso ocorram falhas em um serviço, a capacidade de recuperá-las rapidamente pode originar percepções positivas acerca da qualidade;

segurança (garantia, firmeza) - a capacidade de cortesia e de conhecimento dos colaboradores. Essa dimensão da qualidade engloba outros fatores como o respeito pelo cliente, a competência, a comunicação e o fato de o colaborador estar realmente interessado no melhor para o cliente;

- $\quad$ empatia - a demonstração de atenção personalizada ao cliente e não padronizada. Essa dimensão inclui características como a sensibilidade, a acessibilidade e o esforço dos colaboradores para atender às necessidades específicas de cada cliente;

- $\quad$ aspectos tangíveis -os aspectos físicos das instalações, a aparência do pessoal, o material, as ferramentas e os equipamentos. É considerada uma evidência tangível da atenção e do cuidado para com os detalhes por parte do prestador do serviço a condição do ambiente (limpeza do local, por exemplo). Pode-se considerar nessa dimensão de avaliação aspectos como a conduta de outros clientes (barulho de outros hóspedes em um hotel, por exemplo).

Parasuraman, Zeithaml e Berry (1988) afirmam que, para assegurar uma boa qualidade na prestação de serviços, é fundamental que as percepções dos clientes excedam suas expectativas. Porém, não basta somente a compreensão da importância do oferecimento de serviços com excelente qualidade: é necessário também atenção a um processo contínuo que possa monitorar as percepções do cliente a respeito da qualidade, para que, assim, sejam adotados os melhores meios para a melhoria.

Dessa forma, em virtude de a $P+L$ e a gestão da qualidade terem impactos semelhantes quando orientadas ao setor de serviços, permitindo mudanças na percepção do cliente, tanto interno quanto externo, e melhoria contínua de atividades e processos, merecem estudos mais aprofundados que explorem tal relação. 


\section{MÉTODO DE PESQUISA}

O método adotado nesta pesquisa é o estudo qualitativo de natureza exploratória (MARCONI; LAKATOS, 2003). Para isso, será apresentada uma associação, baseada na literatura, entre a metodologia de implantação da Produção Mais Limpa (P+L) (UNEP/UNIDO, 1991) e as cinco dimensões da qualidade em serviços (PARASURAMAN; ZEITHAML; BERRY, 1988). A metodologia UNEP/UNIDO (1991) é composta de cinco etapas: (A) planejamento e organização, (B) pré-avaliação, (C) avaliação, (D) estudos de viabilidade e (E) implementação e monitoramento (UNEP/UNIDO, 1991; MASSOTE; SANTI, 2013). Esses estágios, por sua vez, são subdivididos, neste trabalho, em 16 passos, como apresentado na Figura 1.

A associação entre as etapas e as dimensões da qualidade foi estabelecida fundamentando-se nas seguintes questões: Quando as etapas e fases da P+L estiverem sendo executadas, há também a abrangência de qual dimensão da qualidade em serviços? Quais as razões para que isso ocorra?

Figura 1 - Etapas e passos da metodologia $\mathrm{P}+\mathrm{L}$



Fonte: adaptado de UNEP/UNIDO (1995).

\section{ANÁLISE DOS RESULTADOS}

A seguir, são apresentadas as etapas da metodologia de implantação da Produção Mais Limpa $(\mathrm{P}+\mathrm{L})$ e a relação entre seus respectivos passos e as dimensões da qualidade. Com essa análise, busca-se discutir as razões e implicações que justificam cada associação.

\subsection{Etapa A: planejamento e organização}

Tendo em vista que as dimensões da qualidade para serviços referem-se ao cumprimento de prazos (confiabilidade), ao pronto atendimento (responsividade), à cortesia (segurança), à atenção personalizada (empatia) e aos aspectos físicos (aspectos tangíveis), percebe-se que elas 
somente serão percebidas na atividade relacionada à "identificação de barreiras e soluções", pois este é o primeiro momento da metodologia que avalia o ambiente organizacional e as relações entre seus atores, ou seja, é a primeira vez que aparece a figura do cliente (cliente interno), elemento fundamental na avaliação da qualidade (DEMING, 1982).

Nessa atividade intitulada "identificação de barreiras e soluções", as cinco dimensões estão relacionadas. Isso pode ser percebido na ligação entre possíveis problemas que dificultam a implementação do programa e, ao mesmo tempo, afetam a prestação do serviço como, por exemplo, conforme UNEP/UNIDO (1995), a dificuldade de obter informações sobre os setores. Por essa dificuldade estar presente, de acordo com os conceitos das dimensões propostas por Parasuraman, Zeithaml e Berry (1985), as dimensões que estão relacionadas são a responsividade, a segurança e a empatia (AGARWAL, MALHOTRA; BOLTON, 2010). Outro exemplo, segundo a UNEP/UNIDO (1995), é a falta de consciência, habilidade técnica e aptidão dos colaboradores, fator que pode impactar a qualidade do serviço; por isso, as dimensões associadas são a confiabilidade e os aspectos tangíveis (AGARWAL; MALHOTRA; BOLTON, 2010)

No passo intitulado "decidir o foco da P+L", que engloba, conforme UNIP/UNIDO (1995), a decisão do escopo (se a implantação da $\mathrm{P}+\mathrm{L}$ incluirá toda a planta ou irá limitar-se a determinadas unidades ou departamentos) e da ênfase (em termos de materiais), deve haver uma avaliação de toda a empresa e das possibilidades de otimização no uso de recursos naturais e na minimização de desperdícios (UNEP/UNIDO, 1991; UNEP/UNIDO, 1995). Essa avaliação também está presente nas práticas de avaliação da qualidade que, em serviços, utiliza como parâmetro as cinco dimensões para identificar aspectos críticos (PARASURAMAN; ZEITHAML; BERRY, 1988). Dessa forma, quando se decide a orientação da $\mathrm{P}+\mathrm{L}$ na empresa, ocorrerá a avaliação de problemas ligados às cinco dimensões.

\subsection{Etapa B: pré-avaliação}

Na segunda etapa de implementação da metodologia de $\mathrm{P}+\mathrm{L}$, as dimensões da qualidade mostram-se envolvidas desde o início do processo. Na atividade de "preparação de informações básicas (diagrama de fluxo)", segundo UNEP/UNIDO (1991, 1995), o ecotime deve listar todas as operações importantes que ocorrem no local onde vai aplicar a $\mathrm{P}+\mathrm{L}$, desde o recebimento das entradas (inputs) até a expedição das saídas (outputs), e, em seguida, esquematizá-las na forma de um diagrama de blocos, que indica a relação entre todas as atividades do processo de serviço.

Esse diagrama permite que uma visão sistêmica do processo ou setor em análise (SLACK; CHAMBERS; JOHNSTON, 2009). Quando se busca a melhoria da qualidade, é imprescindível o conhecimento e a avaliação das inter-relações existentes entre os processos internos da organização, pois permite uma compreensão mais acurada do grau de influência de uma atividade em relação à atividade subsequente e assim por diante (SLACK; CHAMBERS; JOHNSTON, 2009). Tal análise possibilita, também, a identificação de possíveis rupturas, pontos de impacto negativo ou desconexões entre as atividades, fatores esses que afetam diretamente a qualidade da prestação do serviço. Dessa maneira, pelas definições de Parasuraman, Zeithaml e Berry (1985), está sendo realizada uma avaliação das seguintes dimensões: confiabilidade, responsividade e aspectos tangíveis, tendo em vista o fato de o diagrama permitir a identificação de pontos de impacto negativo no tempo do serviço (confiabilidade), na agilidade do processo (responsividade) e na utilização de recursos (aspectos tangíveis) (PARASURAMAN; ZEITHAML; BERRY, 1988; AGARWAL; MALHOTRA; BOLTON, 2010).

Já na "preparação do ecomapa", o objetivo consiste na captação das observações feitas no passo anterior (construção do diagrama do fluxo), principalmente no que diz respeito ao 
layout da empresa ou do setor em questão (UNEP/UNIDO, 1991; UNEP/UNIDO, 1995; CETESB/ UNEP, 2002). O ecomapa, segundo UNEP/UNIDO (1995), é um indicador direto de organização e arrumação, podendo ser desenvolvido para temas específicos, como consumo de energia, geração de resíduos, segurança e riscos ambientais (UNEP/UNIDO, 1995).

Vale ressaltar que um estudo voltado ao layout objetiva minimizar o investimento em equipamentos, minimizar o tempo do processo, utilizar o espaço de uma forma mais eficiente, promover segurança para os funcionários nos postos de trabalho, flexibilizar as operações, reduzir os custos com a utilização de materiais e melhorar a estrutura da empresa como um todo. Em outras palavras, busca-se reduzir ou eliminar perdas durante as operações relativas à movimentação desnecessária, ao retrabalho, a filas e a estoques (SLACK; CHAMBERS; JOHNSTON, 2009), fatores ligados à qualidade em serviços nas dimensões confiabilidade, responsividade e aspectos tangíveis (PARASURAMAN; ZEITHAML; BERRY, 1988; AGARWAL; MALHOTRA; BOLTON, 2010).

Já na "preparação do balanço de materiais", que compreende, segundo UNEP/UNIDO (1991, 1995), à realização de um inventário de materiais utilizados nas operações dos serviços, esta etapa se relaciona com a dimensão "aspectos tangíveis", visto que busca analisar todos os recursos que podem gerar resíduos (PARASURAMAN; ZEITHAML; BERRY, 1985; UNEP/UNIDO, 1995).

\subsection{Etapa C: avaliação}

O primeiro passo dessa etapa é "identificar causas de problemas e diagnósticos". Aqui o ecotime deve começar a gerar alternativas de ações de P+L (UNEP/UNIDO, 1995) por meio da metodologia UNEP/UNIDO chamada de "diagnóstico de causa". Para isso, sugere-se a utilização da ferramenta da qualidade conhecida como "espinha de peixe". Essa ferramenta permite a visualização da sequência de tarefas relacionadas ao serviço, desde a preparação (set-up) até sua finalização (ISHIKAWA, 1990). Para o uso dessa ferramenta, é necessária uma avaliação, na empresa de serviços, de todas as dimensões da qualidade, pois só assim haverá uma compreensão significativa das razões por trás dos problemas e, consequentemente, dos desperdícios relacionados a eles (ISHIKAWA, 1990; SILVA et al., 2013).

Da mesma forma, os dois passos seguintes, intitulados "geração de opções (brainstorming)" e "seleção de opções", também incluirão todas as dimensões da qualidade. O brainstorming refere-se à emissão de idéias de forma livre por um determinado grupo, em um ambiente livre de críticas e de restrições à imaginação, em um curto espaço de tempo (ISAKSEN; GAULIN, 2005). Essas fases têm por base as análises feitas anteriormente no passo "identificação das causas e diagnósticos", que são o ponto de partida para o exercício da reflexão (UNEP/UNIDO, 1991; UNEP/UNIDO 1995; CETESB/UNEP, 2002). Portanto, devem ser considerados todos os possíveis aspectos ligados às cinco dimensões da qualidade (PARASURAMAN; ZEITHAML; BERRY, 1985; AGARWAL; MALHOTRA; BOLTON, 2010), para que as soluções permitam a realização de "serviços mais limpos" e da forma mais eficiente possível (GLAVIC; LUKMAN, 2007).

\subsection{Etapa D: estudos de viabilidade}

Essa etapa, constituída dos passos "avaliação técnica, econômica e ambiental" e "selecionar opções viáveis", está totalmente integrada com as dimensões da qualidade em serviços. Os resultados obtidos nessa fase da metodologia permitirão avaliar e selecionar as medidas mais viáveis de $\mathrm{P}+\mathrm{L}$ ou dos chamados serviços mais limpos (GLAVIC; LUKMAN, 2007).

No estudo técnico, econômico e ambiental, consideram-se fatores como: (a) impacto 
da possível alteração da operação no processo; (b) verificação dos funcionários e departamentos atingidos pelas mudanças; (c) alterações necessárias em termos de pessoal e operações; (d) redução, em termos quantitativos, dos materiais e da energia; (e) investimento necessário e custos operacionais; e (f) economia que virá com a mudança. Juntamente com essas avaliações, como se trata do setor de serviços, deve-se também verificar o que, na percepção do cliente, possíveis mudanças nos aspectos relacionados a cada uma das cinco dimensões podem trazer de benefícios, tais como: alterações no tempo de execução do serviço (confiabilidade), mudanças na inter-relação das pessoas e das atividades organizacionais (responsividade, segurança e empatia) e modificações físicas na empresa (aspectos tangíveis) (PARASURAMAN; ZEITHAML; BERRY, 1985; FITZSIMMONS; FITZSIMMONS, 2007; AGARWAL; MALHOTRA; BOLTON, 2010).

\subsection{Etapa E: implementação e monitoramento}

Nessa etapa, pode-se verificar um diálogo com as dimensões no passo intitulado "sustentação e avaliação da $\mathrm{P}+\mathrm{L}$ ". É necessário que um sistema de indicadores seja mantido para monitorar as alterações feitas (UNEP/UNIDO, 1991; UNEP/UNIDO, 1995; CETESB/UNEP, 2002) e, principalmente, a percepção do cliente quanto ao serviço (PARASURAMAN; ZEITHAML; BERRY, 1988; AGARWAL; MALHOTRA; BOLTON, 2010), pois só assim será possível identificar a necessidade de novas alterações que venham a modificar a postura ambiental da empresa pela realização de serviços mais limpos e a forma como os clientes internos percebem a dinâmica organizacional.

Por fim, para que haja uma visualização da totalidade das relações encontradas neste estudo, foi esquematizada, na Tabela 1, a associação entre a metodologia em questão e as dimensões da qualidade em serviços.

Tabela 1 - Associação entre a metodologia e as dimensões da qualidade



Fonte: elaborada pelos autores. 


\section{CONSIDERAÇÕES FINAIS}

O presente trabalho teve como objetivo analisar a relação entre a Produção Mais Limpa $(\mathrm{P}+\mathrm{L})$ e o setor de serviços. Para isso, foi apresentada uma associação, baseada na literatura, entre a metodologia de implantação da P+L (UNEP/UNIDO, 1991) e as cinco dimensões da qualidade utilizadas pelo setor de serviços como forma de verificar impactos na percepção dos clientes quanto a melhorias nas operações e nos processos (PARASURAMAN; ZEITHAML; BERRY, 1988; FITZSIMMONS; FITZSIMONS, 2007).

Embora existam dificuldades na visualização da $\mathrm{P}+\mathrm{L}$ em serviços, essa prática pode ser adotada para promover ações ambientais por meio de serviços mais limpos. Isso pode ser verificado através das possíveis melhorias trazidas para o cliente interno pela aplicação da metodologia em aspectos ligados à confiabilidade, à responsividade, à segurança, à empatia e a aspectos tangíveis, que compõem as dimensões da qualidade em serviços de Parasuraman, Zeithaml e Berry (1988).

Portanto, é coerente afirmar que a $\mathrm{P}+\mathrm{L}$ não deve se restringir ao setor manufatureiro, uma vez que traz ao setor de serviços práticas que aumentem a ecoeficiência e tornam mais eficazes as relações internas à organização, como a valorização do meio ambiente e o aumento da satisfação dos clientes internos (colaboradores). Em suma, foi identificada a existência de inter-relação entre o setor terciário e a P+L, pois, quando as etapas da metodologia são executadas, há influência sobre as dimensões da qualidade. Esse impacto acarreta benefícios não só em questões ambientais, mas na eficiência dos processos e na melhoria da percepção do cliente quanto ao serviço prestado.

Como contribuição, este estudo deu um passo a mais na consolidação do relacionamento entre $\mathrm{P}+\mathrm{L}$ e o setor de serviços. Compreender melhor a relação entre esses dois temas é útil para a internalização dos benefícios e de novas ações sustentáveis para o setor. Além disso, a compreensão por parte dos empresários e gestores de que a relação existe possibilita a aplicação prática de técnicas mais incisivas e eficientes no ambiente organizacional.

Entretanto, é relevante destacar que este estudo apresenta algumas limitações que poderão ser posteriormente trabalhadas e sanadas. A principal delas consiste no fato de que a relação proposta não foi verificada em campo, o que possibilitaria comprovar que a $P+L$ realmente melhora a percepção do cliente interno quanto à qualidade do serviço.

Por fim, são necessárias novas pesquisas, que visem apresentar contribuições e encontrar maneiras de intensificar a divulgação dos conceitos e das práticas de $\mathrm{P}+\mathrm{L}$ no setor terciário. Uma delas poderia ser a verificação empírica da relação aqui proposta para confirmar e avaliar a sua adequação.

\section{REFERÊNCIAS}

AGARWAL, J., MALHOTRA, N. K., BOLTON, R. N. $A$ cross-national and cross-cultural approach to global market segmentation: an application using consumers' perceived service quality, Journal of International Marketing, v. 18, n. 3, 2010, p. 18-40, 2010.

BHAT, M. A. Tourism service quality: a dimension-specific assessment of SERVQUAL, Global Business Review, v. 13, n. 2, p. 327-337, 2012.

\section{CAMBRIDGE. Cambridge advanced learner's dictionary, 2013.}

CETESB/UNEP. Status report: cleaner production in Latin America and the Caribbean. CETESB, UNEP, Paris, 2002.

DEMING, W. E. Quality, productivity and competitive position. Boston: MIT Press, 1982.

FITZSIMMONS, J. A., FITZSIMMONS $M$. Administração de Serviços: Operações, Estratégia e Tecnologia de Informação. Porto Alegre: Bookman, 2007. 
FRESNER, J., JANTSCHGI, J., BIRKEL, S., BARNTHALER, J., KRENN, C. The theory of inventive problem solving (TRIZ) as option generation tool within cleaner production projects, Journal of Cleaner Production, v. 18. p. 128-136, 2010.

GLAVIC, P., LUKMAN, R. Review of sustainability terms and their definition. Journal of Cleaner Production, v. 15. p. 1875-1885, 2007.

ISAKSEN, S. G., GAULIN, J. P. A reexamination of brainstorming research: implications for research and practice. Gifted Child Quartely. v. 49. n. 4. p. 315-329, 2005

ISHIKAWA, K. Introduction to quality control, 3A Corporation. Tokyo, 1990.

MARCONI, M. A.; LAKATOS, E. M. Fundamentos de metodologia científica. São Paulo: Atlas, 2003.

MASSOTE, C. H. R., SANTI, A. M. M. Implementation of a cleaner production program in a brazilian wooden furniture factory. Journal of Cleaner Production, v. 46. p. 89-97, 2013.

MDIC, Comércio de serviços: informações gerais, exportações e como notificar barreiras. Disponível em: <http://www.desenvolvimento. gov.br/arquivo/secex/bartecnicas/barservicos/ cartilhaservicos.pdf>. Acesso em: 09 jan. 2014.

MICHAELIS. Dicionário. Editora Melhoramentos Ltda, 2009.

PARASURAMAN, A., ZEITHAML, V.A. AND BERRY, L.L. A conceptual model of service quality and its implications for future research, Journal of Marketing. v. 49, p. 41-50, 1985.

PARASURAMAN, A., ZEITHAML, V.A., BERRY, L.L. SERVQUAL: a multiple-item scale for measuring consumer perceptions of service quality, Journal of Retailing, v. 64 n. 1, p. 1240, 1988.
REYNOLDS, N., SMITH, A. Assessing the impact of response styles on cross-cultural service quality evaluation: a simplified approach to eliminating the problem. Journal of Service Research, v. 13 n. 2, p. 230-243, 2010.

SILVA, D. A. L., DELAI, I., CASTRO, M. A. S., OMETTO, A. Quality tools applied to cleaner production programs: a first approach toward a new methodology. Journal of Cleaner Production, v. 47. p. 89-97, 2013.

SILVA, G. C. S., MEDEIROS, D. D. Metodologia de checkland aplicada à implementação da produção mais limpa em serviços. Gestão \& Produção, v.13, n.3, p.411-422, 2006.

SLACK, N.; CHAMBERS, S., JOHNSTON, R. Administração da produção. São Paulo: Atlas, 2009.

TACHIZAWA, T. Gestão ambiental e responsabilidade social corporativa: estratégias de negócios focadas para a realidade brasileira. São Paulo: Atlas, 2004.

UNIDO/UNEP Audit and reduction manual for industrial emissions and waste. publication. Technical Report Series, n. 7. United Nations Publication, Paris, 1991.

UNIDO/UNEP Guidance materials for the UNIDO/UNEP national cleaner production centres. United Nations Environment Programme, Paris, France, 1995. 